\section{Clasificación anatómica: una propuesta para categorizar las fisuras labiopalatinas}

\section{Anatomical classification: a proposal to categorize cleft lip and palate}

\section{Resumen}

El origen de una clasificación para las fisuras labiopalatinas se remonta desde comienzos del siglo XX. Muchos de los sistemas propuestos por diversos autores no han prosperado, principalmente debido a su complejidad para memorizar y registrar. Esta nota científica resume las clasificaciones de fisuras labiopalatinas más populares entre los profesionales de la salud y además propone una nueva, basada en aspectos anatómicos y morfológicos de las fisuras en los pacientes, la Clasificación Anatómica. Esta clasificación, diseñada y utilizada por la Unidad de Malformaciones Craneofaciales de la Universidad de Chile, busca entregar una solución a esta problemática, categorizando las fisuras, de manera simple y descriptiva, según los tejidos comprometidos (labio, paladar, labiopalatinas), si su manifestación clínica es poco prevalente (atípicas) o si se encuentran asociadas a algún síndrome (sindrómicas).

Palabras clave: Fisura del paladar; Labio leporino; Clasificación (fuente: DeCS BIREME).
Nota Científica

Noemí Leiva ${ }^{1, a, b}$, Carolina Stange ${ }^{1, a}$, Francisca Ayala ${ }^{1, a}$ Vanessa Fuentes $1, a$

${ }^{1}$ Universidad de Chile, Facultad de Odontología, Unidad de Malformación Cráneofacial, Santiago, Chile.

${ }^{a}$ Cirujano Dentista.

${ }^{\mathrm{b}}$ Especialista en Ortodoncia y Ortopedia Maxilofacial.

\section{Correspondencia:}

Carolina Stange

Correo electrónico: carostange@hotmail.com

General Holley 2381, oficina 606 torre B, Providencia,

Santiago, Chile.

\section{Coautores: \\ Noemi Leiva \\ leivanoemi@yahoo.com \\ Francisca Ayala \\ francisca@ayalajimenez.com \\ Vanessa Fuentes \\ vane.fuentes.f@gmail.com}

\section{Editora:}

Dahiana Alfaro Carballido

Universidad de San Martín de Porres, Perú.

Conflicto de intereses: los autores declaran no tener conflictos de interés.

Fuente de financiamiento: autofinanciado.

Recibido: 15/04/2019

Aceptado: 07/06/2019

Publicado: 12/09/2019 


\section{Introducción}

Las fisuras labiopalatinas son una serie de malformaciones que ocurren como consecuencia de la alteración en la fusión de los procesos embriológicos que conforman la cavidad nasal y oral, entre la cuarta y sexta semana de gestación ${ }^{1}$. A nivel mundial se presentan en 1 de cada 700 recién nacidos $(\mathrm{RN})$ vivos, convirtiéndose en una de las alteraciones craneofaciales más prevalentes. En Chile, los valores son bastante similares, describiéndose una prevalencia de 0,7 por $1000 \mathrm{RN}$ vivos para las fisuras de paladar y de 1,4 por $1000 \mathrm{RN}$ vivos, para la fisura de labio ${ }^{2}$.

Aunque su etiología no ha podido ser determinada, se describe que es de tipo multifactorial, influenciada por factores genéticos y factores ambientales (tabaquismo, alcohol, etc.). Los pacientes con esta condición presentan problemas a nivel óseo, muscular, dentoalveolar, de alimentación, fonético e incluso psicológico. En estos casos, el abordaje terapéutico interdisciplinario es fundamental para lograr la correcta rehabilitación de los pacientes y se debe iniciar desde el nacimiento ${ }^{2}$.

Existe una gran variabilidad en la expresión clínica de las fisuras; dependiendo de las estructuras anatómicas que comprometen y la severidad de estas. Es muy importante caracterizar adecuadamente cada caso, ya que esto determina su diagnóstico, entrega una guía para su tratamiento y su posible pronóstico.

Las clasificaciones son una forma de organizar la información, de tal forma que sea más comprensible y clara; permitiendo que se desarrolle una comunicación efectiva entre los distintos profesionales de la salud. En relación a las fisuras labio palatinas, muchas clasificaciones se han propuesto a lo largo de la historia ${ }^{3,4}$, dividiéndose en dos grandes grupos: las basadas en principios embriológicos de las fisuras y las basadas en sus aspectos morfológicos. Sin embargo, al ser fenotípicamente tan diversas, es difícil crear una clasificación precisa, por ende no existe aún un consenso por parte de los clínicos con respecto a cuál utilizar.

La Unidad de Malformaciones Craneofaciales (UMCF) de la Universidad de Chile, anteriormente llamada IRMADEMA (Instituto de Rehabilitación de Malformaciones y Deformaciones Máxilo- Faciales), es una institución chilena que trabaja con pacientes fisurados hace más de 30 años ${ }^{5}$. El propósito de este trabajo es revisar algunas de las clasificaciones de fisuras labiopalatinas más comunes a nivel internacional y proponer un sistema de clasificación que ha desarrollado y utilizado el equipo de ortodoncistas de la UMCF para categorizar a los pacientes a lo largo de estos años, basada en aspectos anatómicos de la morfología de la fisura. Es un sistema simple y descriptivo, el cual se ha denominado Clasificación anatómica de las fisuras labiopalatinas.

\section{Clasificaciones de las fisuras labiopalatinas}

La clasificación de las fisuras labiopalatinas busca ser una herramienta que le permita al equipo multidisciplinario hablar un idioma común, facilitando el diagnóstico, tra- tamiento y la investigación. En el último siglo, se han desarrollado muchos intentos para categorizar las fisuras, muchos de estos sistemas sin mucho éxito, debido a su complejidad para registrar y dificultad para memorizar cada categoría. A continuación describimos algunas de las más clásicas y utilizadas por los profesionales de la salud, así como la propuesta por nuestro equipo de trabajo.

\section{Clasificación de Davis y Ritchie (1922) ${ }^{6}$}

Fue una de las primeras propuestas para categorizar las fisuras. Con un enfoque más quirúrgico, este sistema las divide en tres grupos de acuerdo a su posición en relación al proceso alveolar. Se debe incluir además el lado y extensión de la anomalía:

- Grupo I o Prealveolares: son las fisuras de labio sin compromiso del proceso alveolar; pueden asociarse o no a fisuras de paladar.

\section{- Unilateral (derecha/izquierda, completa/incompleta)}

- Bilateral (derecha completa/incompleta, izquierda completa/incompleta)

- Medianas (menos prevalente, completa/incompleta)

- Grupo II o Postalveolares: fisuras de paladar que no se extienden hacia el proceso alveolar. Pueden asociarse o no a fisuras de labio.

- Paladar blando: la fisura puede abarcar desde solo la úvula hasta el paladar blando completo.

- Paladar duro: se puede manifestar como una muesca o extenderse al agujero incisivo y comprometer paladar blando

- Grupo III o Alveolares: usualmente se asocia a fisura de labio, paladar o ambas.

- Unilateral (derecha/izquierda, completa/incompleta)

- Bilateral (derecha completa/incompleta, izquierda completa/incompleta)

- Medianas (menos prevalente, completa/incompleta)

\section{Clasificación de Veau (1931) ${ }^{3,5}$}

Es una de las clasificaciones más utilizadas, debido a su simplicidad y relevancia clínica; divide a las fisuras según aspectos morfológicos en cuatro grandes grupos:

- Grupo I: fisuras del paladar blando; no contempla aquellas que dividen a la úvula de manera parcial.

- Grupo II: fisuras de paladar duro y blando, que pueden extenderse entre el agujero incisivo y borde posterior del paladar. Describe divisiones de bóveda y velo del paladar, sin comprometer proceso alveolar ni labio.

- Grupo III: fisuras de paladar blando y duro que se extiendan unilateralmente a través del proceso alveolar (unilateral completa). El rasgo de fisura se encuentra entre canino e incisivo lateral, hay compromiso de úvula y el segmento no afectado se une al septum nasal. 
- Grupo IV: fisuras de paladar blando y duro que se extiendan bilateralmente a través del proceso alveolar (bilateral completa). Existe compromiso de úvula y segmentos maxilares no se encuentran unidos al septum nasal.

\section{Clasificación de Harkins (1962) ${ }^{7}$}

Propuesta por Harkins y sus colaboradores en la Asociación Americana de Paladar Fisurado. Es un sistema de clasificación integral, el cual segmenta anatómicamente las fisuras en cuatro grupos; debiéndose describir además la ubicación (izquierda, derecha, línea media), extensión $(1 / 3,2 / 3,3 / 3)$ y ancho de la fisura

- Fisuras del prepaladar: pueden ser uni o bilaterales. Su límite posterior corresponde al agujero incisivo.

- Fisuras de Labio

- Fisura alveolar

- Fisura de labio, alveolo y paladar primario

- Fisuras del paladar: solo en línea media. Su límite anterior corresponde al agujero incisivo.

- Fisuras de paladar blando

- Fisura de paladar duro

- Fisura de paladar blando y duro

- Fisuras del prepaladar y paladar (combinación de cualquier variación de fisuras prepalatinas y palatinas).

- Fisuras orofaciales (menos prevalentes).

- Fisura del proceso mandibular

- Fisura naso-ocular

- Fisura oro-ocular

- Fisura oroaural

\section{Clasificación de Kernahan (1971) ${ }^{8}$}

Kernahan conjuntamente con Stark (1958) realizaron una clasificación proponiendo el uso de un diagrama en Y que permitiera esquematizar las fisuras con respecto a su ubicación y extensión, facilitando el registro y la comprensión clínica. Este esquema representa una fisura bilateral completa, y de acuerdo a las estructuras comprometidas en el paciente, el clínico solo debe marcar el número correspondiente para registrarlo en la ficha. En 1998, Smith et al ${ }^{9}$ realizaron una modificación de este esquema; permitiendo detallar más específicamente las fisuras que comprometen labio, incorporando el agujero incisivo como límite entre paladar anterior y posterior y añadiendo un ítem para fisuras submucosas. A saber (Figura 1):

- Defectos que se encuentren al lado derecho serán descritos con el número correspondiente y si se encuentran al lado izquierdo se debe agregar una comilla.

- El número 1 representa el labio, el cual se divide en tercios mediante las letras a, b, c según extensión de la fisura y además se agrega la letra $\mathrm{d}$ para la banda de Simonart.
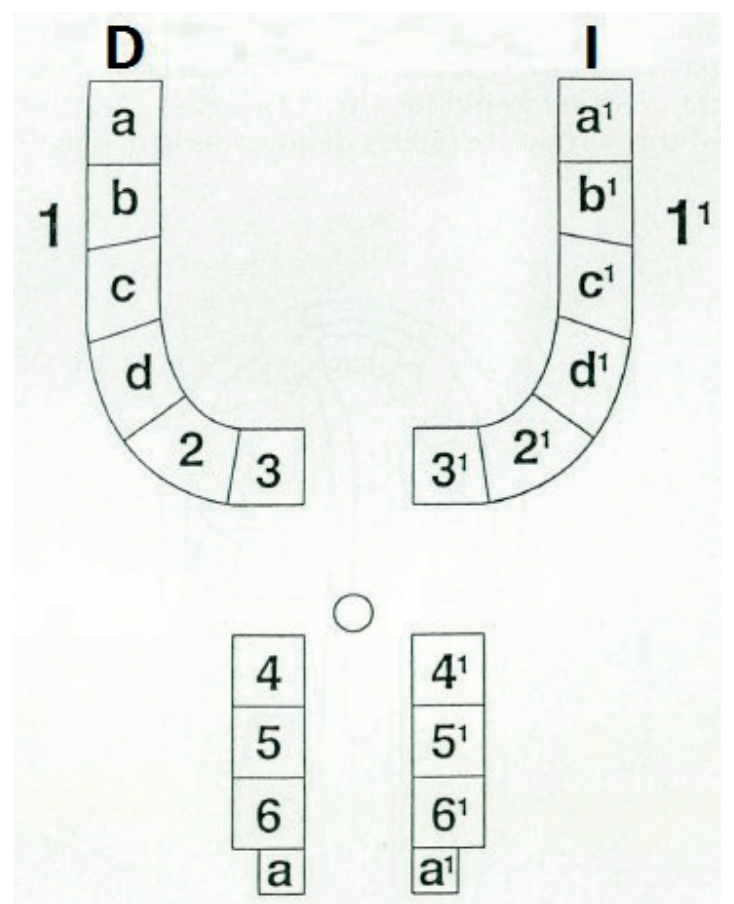

Figura 1. Diagrama de Kernahan, modificado por Smith ${ }^{9}$

- El número 2 representa al proceso alveolar.

- El número 3 representa la porción más anterior del paladar duro (premaxila).

- El número 4 representa el paladar duro, pero hasta hueso maxilar.

- El número 5 representa el paladar duro, incluyendo hueso palatino.

- El número 6 representa el paladar blando.

- La letra a representa las fisuras submucosas.

\section{Clasificación LAHSAL (2005) ${ }^{10}$}

Modificación realizada por el Royal College of Surgeons de Inglaterra a la clasificación propuesta por Kriens en 1987 (LAHSHAL). El nombre corresponde a las siglas de las áreas anatómicas afectadas por la fisura (Figura 2):

- L: labio derecho (lip)

- A: proceso alveolar derecho (alveolus)

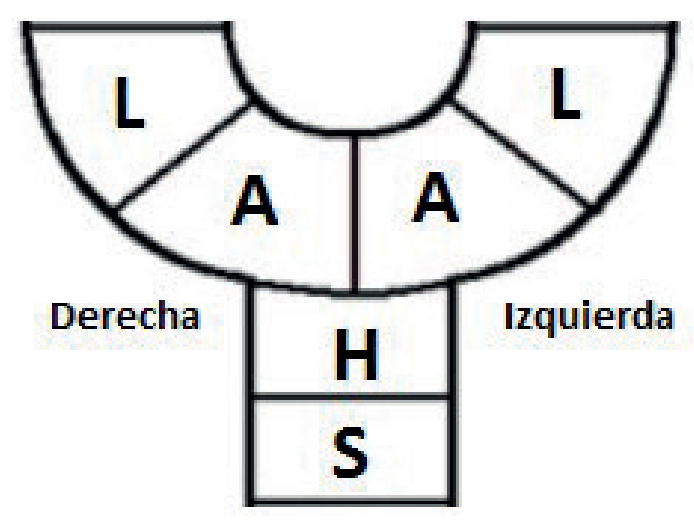

Figura 2. Diagrama de LAHSAL $^{5}$ 
- H: paladar duro (hard palate)

- S: paladar blando (soft palate)

- A: proceso alveolar izquierdo

- L: labio izquierdo

Cuando corresponde a una fisura completa, debe registrarse en la ficha en letra mayúscula si es bilateral (LAHSAL) y minúscula si es unilateral (lahsal). En caso de que alguna estructura no estuviese comprometida, entonces se representa con un punto [.]. Algunos ejemplos serían:

- Fisura completa de labio derecho: L.....

- Fisura incompleta de labio y alveolo izquierdo: ....al

\section{Clasificación Anatómica de la UMCF (2004) ${ }^{5}$}

Basado en los principios de la clasificación de Harkins, categoriza las fisuras morfológicamente, según su ubicación anatómica. Con un objetivo más bien diagnóstico, la clasificación anatómica es un sistema simple y de mucha utilidad para el clínico en su práctica con niños de diferentes edades, ya que es conciso y descriptivo. Inicialmente dividía a las fisuras en cuatro categorías, pero actualmente se ha agregado una quinta. Cuando las fisuras son unilaterales, se debe señalar el lado afectado. A saber (Tabla).

\section{Fisuras de labio}

- Muesca: mínima expresión de la fisura que afecta solo la porción muscular del labio.

- Labio fisurado incompleto: abarca todo el grosor del labio, sin comprometer base de nariz.

- Labio fisurado completo: abarca todo el grosor del labio, llegando a base de nariz y distorsionando el ala. También compromete piso de cavidad nasal.
- Fisura de labio y reborde alveolar unilateral: compromete paladar primario hasta el agujero incisivo.

- Fisura de labio y reborde alveolar bilateral: compromete paladar primario hasta el agujero incisivo.

\section{Fisuras de paladar}

- Paladar duro: puede abarcar desde el agujero incisivo hasta la el borde posterior de la lámina horizontal del hueso palatino.

- Paladar blando: afecta solo zona muscular del paladar.

- Fisura de paladar duro y blando: cuando coexisten.

- Fisura submucosa: no existe unión ósea ni muscular del paladar, solo mucosa.

- Úvula bífida: división mediana localizada en la úvula.

- Fisura submucosa y úvula bífida, cuando coexisten.

\section{Fisuras de labio y paladar}

- Fisura de labio y reborde alveolar unilateral complicado con fisura velopalatina (FVP).

- Fisura de labio y reborde alveolar bilateral complicado con fisura velopalatina.

\section{Fisuras atípicas}

- Fisuras poco prevalentes, que no entran en las categorías anteriores, ya que no siguen el recorrido anatómico habitual y presentan expresiones clínicas muy variadas.

\section{Fisuras sindrómicas}

- Se refiere a aquellas fisuras congénitas que no se manifiestan de manera aislada, sino que se encuentran

Tabla. Cuadro de registro basado en la clasificación anatómica, utilizado en la ficha clínica de la UMCF ${ }^{5}$

1. Diagnóstico (encerrar opción)

A.1 Muesca de labio izquierda.

B.1 Muesca de labio derecha.

A.2 Fisura de labio incompleta

B.2 Fisura de labio incompleta izquierdo.

derecha.

A.3 Fisura de labio completa

B.3 Fisura de labio completa derecha. izquierdo.

A.4 Fisura de labio y reborde alveolar izquierdo.

A.5 Fisura de labio y reborde alveolar derecho.

A.6 Fisura de labio y reborde alveolar bilateral.

A.7 Fisura de labio y reborde alveolar derecho complicado con FVP.

A.8 Fisura de labio y reborde alveolar unilateral izquierdo complicado con FVP.

A.9 Fisura de labio y reborde alveolar bilateral complicado con FVP.

A.10 Fisura de paladar duro.

A.11 Fisura de paladar blando.

A.12 Fisura de paladar duro y blando.

A.13 Fisura submucosa.

A.14 Úvula bífida.

A.15 Fisura submucosa y úvula bífida.

A.16 Atípicas.

A.17 Sindrómicas 
asociadas a algún síndrome cráneofacial. Se debe describir la fisura según las estructuras anatómicas asociadas, de la forma señalada previamente.

En la ficha clínica diseñada y utilizada por la UMCF, existe una sección con las fisuras recién descritas a modo de alternativas, para poder facilitar su registro, tal como se observa en la tabla. En el apartado original de la ficha se utiliza el término labio leporino, el cual ha sido reemplazado en el cuadro de ejemplo por el de labio fisurado. Ambos términos son considerados por parte de nuestro equipo como sinónimos, debido principalmente a que en Chile, el concepto de labio leporino es aún utilizado por un gran porcentaje de profesionales de la salud; además es parte de los descriptores en Ciencias de la Salud (DeCS) de BIREME, por ende es importante estar familiarizado con ambos. La experiencia clínica utilizando esta clasificación para las fisuras craneofaciales no solo se limita a la nuestra por parte del equipo de Ortodoncia de la UMCF. El servicio de cirugía máxilofacial del Hospital San Borja Arriarán, en Santiago de Chile, ha utilizado exitosamente la Clasificación Anatómica con sus pacientes desde la instauración del plan AUGE en 2005, tanto para fines diagnósticos, como de investigación ${ }^{1,11}$.

\section{Conclusiones}

Es difícil lograr un consenso con respecto a que clasificación utilizar para las fisuras labiopalatinas, desafortunadamente ningún sistema único ha sido universalmente aceptado y utilizado. Los clínicos a menudo recurren a términos descriptivos y esquemas para proporcionar la especificidad requerida en casos individuales.

En la literatura existen más 30 tipos de clasificaciones ${ }^{4}$, cada una con sus aspectos positivos y negativos. Conscientes de aquello y sin la intención de añadir una clasificación más a la lista pre-existente, proponemos este sistema basado en el éxito de nuestra experiencia clínica, en especial cuando se trabaja con un equipo multidisciplinario. Las ventajas que observamos de la clasificación anatómica son que facilita la comunicación interprofesional, al ser un sistema simple y con conceptos manejables por el clínico y facilita su registro en la ficha clínica, al ser cada categoría descriptiva en si misma con respecto al tipo de fisura que posee el paciente.

Si bien este sistema no incluye extensión ni severidad de la fisura, como otros, la ficha clínica diseñada y utilizada por la UMCF posee un apartado a completar en donde se puede describir características más específicas con respecto a fisura del paciente.

Se aprecian los comentarios y sugerencias de los lectores y usuarios de la presente clasificación.

\section{Referencias bibliográficas}

1. Cordero CE, Correa HS, Pantoja PR. Prevalence of patients with cleft lip and palate who were treated at San Borja Arriarán Clinical Hospital in Santiago de Chile, within the AUGE Healthcare Plan. Int J Odontostomat. 2015;9(3):469-473. DOI: $10.4067 / S 0718$ $381 X 2015000300018$.

2. MINISTERIO DE SALUD. Guía Clínica AUGE Fisura Labiopalatina. 3a ed. Santiago de Chile, Ministerio de Salud (Minsal), 2015. [Internet ] [citado 2017 Mayo 13] Disponible en: http://www.bibliotecaminsal.cl/wp/ wp-content/uploads/2016/04/guia-FisuraLabioPalatina-2015-CM.pdf.

3. Allori AC, Mulliken JB, Meara JG, Shusterman S, Marcus JR. Classification of cleft lip/palate: then and now. Cleft Palate Craniofac J. 2017;54(2):175-188. DOI: 10.1597/14-080.

4. Shankar C. Classification of Cleft Lip and Palate. Researchgate. 2018. [Internet] [consultado 10 de Abril 2019] Disponible en: https://www.researchgate.net/profile/ Chetan_Shankar/publication/327013345_Classification_of_Cleft_Lip_and_Palate/links/5b72a788299bf14c6da1a0a8/Classification-of-Cleft-Lip-and-Palate. pdf?origin=publication_detail.

5. Muñoz A, Cauvi D, Leiva N. Clasificación de las fisuras labio palatinas. En: Cauvi D, Leiva N, editoras. Etiopatogenia y tratamiento de las fisuras Labio-Maxilo-Palatinas. $2^{a}$ ed. Santiago de Chile: Facultad de Odontología Universidad de Chile; 2004. p 81-96.

6. Davis JS, Ritchie HP. Classification of congenital clefts of the lip and palate with a suggestion for recording these cases. JAMA. 1922;79(16):1323-1327.

7. Harkins CS, Berlin A, Harding RL, Longacre JJ, Snodgrasse RM. A Classification of Cleft Lip and Cleft Palate. Plast Reconstr Surg. 1962;29(1):31-39.DOI: http://dx. doi.org/10.1097/00006534-196201000-00005.

8. Kernahan DA. The striped $\mathrm{Y}$ a symbolic classification for cleft lips and palate. Plast Reconstr Surg. 1971;47(5):469470. DOI: 10.1097/00006534-197105000-00010.

9. Smith AW, Khoo AK, Jackson IT. A modification of the Kernahan "Y" classification in cleft lip and palate deformities. Plast Reconstr Surg. 1998;102(6):1842-1847. DOI: 10.1097/00006534-199811000-00005.

10. Shah SN, Khalid M, Khan MS. A review of classification systems for cleft lip and palate patients. Morphological classifications. JKCD. 2011;1(2):95-99.

11. Martínez G. Perfil epidemiológico de los pacientes con fisuras labio-máxilo-palatina no sindrómicas atendidos en el Hospital San Borja Arriarán durante el período 2005-2015. Santiago, Chile: Universidad de Chile - Facultad de Odontología; 2017. [Internet] [consultado 30 de Marzo 2019]. Disponible en: http://repositorio.uchile.cl/handle/2250/146567. 
